\title{
Can radiotherapy be a viable salvage treatment option for the relapsed seminoma confined to the infra-diaphragm region recurring after primary chemotherapy for bulky stage II seminoma?
}

\author{
Richard Choo, MD; ; Fernando Quevedo, MD; ${ }^{\dagger}$ Christopher S. Choo, ${ }^{*}$ Michael Blute, MD
}

\begin{abstract}
There has been a paucity of research describing a potential role of radiotherapy as salvage treatment for recurrent seminoma following primary chemotherapy for bulky stage IIC seminoma. We report a case of a bulky stage IIC seminoma relapsed in the pelvis after primary chemotherapy and surgery for post-chemotherapy residual mass, which was subsequently salvaged with radiotherapy. The patient has remained free of relapse at 3.7 years post-salvage radiotherapy. This case demonstrates that radiotherapy can be a salvage therapeutic option for recurrent seminoma following primary chemotherapy for bulky stage IIC seminoma, provided that the recurrent tumour is confined to a limited area of the infradiaphragmatic region. There is a need for further study to examine the potential role of radiotherapy as a salvage therapeutic tool for post-chemotherapy recurrent seminoma.
\end{abstract}

Can Urol Assoc J 2010;4(5):E137-140

\section{Introduction}

Testicular seminoma is highly curable, and exhibits a high sensitivity to both radiotherapy and cisplatin-based chemotherapy. For testicular seminoma, radiotherapy is typically applied in the following clinical situations: (1) In Stage I, radiotherapy is applied as an adjuvant treatment following a radical inguinal orchiectomy to minimize a relapse in the retroperitoneum and/or ipsilateral pelvis; and (2) In Stage IIA and IIB where regional lymph nodal metastasis is less than $5 \mathrm{~cm}$, radiotherapy is given as a definitive therapeutic intervention. In Stage IIC where regional node metastasis exceeds $5 \mathrm{~cm}$, most clinicians use cisplatin-based combination chemotherapy as the primary treatment. There has been little research describing the potential role of radiotherapy as salvage treatment for recurrent seminoma following primary chemotherapy for bulky stage IIC seminoma. We report a case of a bulky stage IIC seminoma relapsed in the pelvis after primary chemotherapy and surgery for post-chemother- apy residual mass, which was subsequently salvaged with radiotherapy to the pelvis and retroperitoneum.

\section{Case report}

In January 2005, a 42-year-old previously healthy man presented with a sudden onset of pain and edema in the left lower extremity. A computed tomography (CT ) scan of the abdomen and pelvis revealed a large mass in the left pelvis measuring $7 \times 7 \times 8 \mathrm{~cm}$ extending along the common iliac vessels. The mass compressed the left common iliac, external iliac and internal iliac veins, and caused moderate hydroureteronephrosis for which he subsequently had a ureteral stent. In addition, there were several small lymph nodes along the left iliac vessels and left retroperitoneum, most of them measuring less than $1 \mathrm{~cm}$. A scrotal ultrasound showed a heterogeneously hypoechoic left testicle containing at least two masses with the largest measuring about $2 \mathrm{~cm}$. The right testicle was normal. A CT scan of the chest was clear with no evidence of metastatic disease. Alpha-fetoprotein was normal, 1 (normal: $<6 \mathrm{ng} / \mathrm{mL}$ ). Beta-human chorionic gonadotropin (HCG) was elevated, 67 (normal: $<0.7 \mathrm{IU} / \mathrm{L}$ ). Lactate dehydrogenase (LDH) was mildly elevated, 246 (normal: 122-222 U/L). His notable past medical history was a left inguinal hernia repair at the age 25 years. On January 25,2005 , he underwent a left radical inguinal orchiectomy. Pathology revealed seminoma forming a multilobulated nodule measuring $3.5 \times 2.3 \times 2 \mathrm{~cm}$. The tumour was confined to the testicle with no invasion into the tunica albuginea. There was no angiolymphatic space involvement. The resection margins including the spermatic cord margin were negative. His seminoma was staged IIC. The pathology was reviewed and confirmed by an expert genitourinary pathologist. The case was discussed at a multidisciplinary tumour board.

He underwent 3 cycles of BEP (bleomycin, etoposide, and cisplatin) chemotherapy, which was completed on March 29, 2005. He had a follow-up CT scan of the abdomen and pelvis on April 11, 2005 (Fig. 1), which revealed a residual mass measuring $3.4 \times 3.8 \mathrm{~cm}$ in the left pelvis at the bifur- 


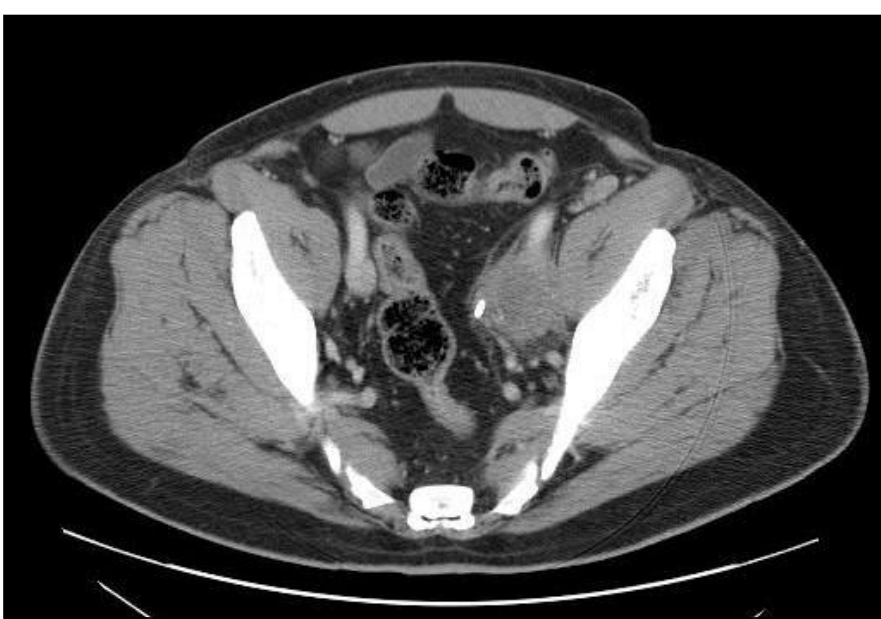

Fig. 1. Residual left pelvic mass after 3 cycles of BEP (bleomycin, etoposide and cisplatin) chemotherapy in April 2005.

cation of the left common iliac vessels. At the time, his beta-HCG returned to normal $(<0.7)$, and so did LDH (180). Alpha-fetoprotein remained normal (1.2). On May 3, 2005, he had a surgical excision of the residual mass in the left pelvis. Pathology revealed a multilobulated mass measuring $5.2 \times 3 \times 2.5 \mathrm{~cm}$, which was a necrotic tissue. There was no viable tumour present. Five other left iliac lymph nodes were negative for metastatic disease.

He had a follow-up CT scan of the chest, abdomen, and pelvis on June 27,2005 . This revealed a small mass measuring $1.5 \times 1.5 \mathrm{~cm}$ in the left pelvis (Fig. 2). The subsequent CT scan of the chest, abdomen and pelvis on October 12, 2005 revealed that the left pelvic mass increased in size, measuring $3.7 \times 4.4 \mathrm{~cm}$ (Fig. 3). There was no other suspicious lymphadenopathy including the retroperitoneum. At the same time, his beta-HCG increased to 7.9, while alphafetoprotein remained normal (0.8). This clinical picture was highly suggestive of the recurrence of seminoma.

He then underwent salvage radiotherapy to the retroperitoneum and the bilateral pelvis. The radiotherapy was completed on November 22, 2005. The radiotherapy consisted of $3060 \mathrm{cGy}$ in 17 fractions to the retroperitoneum and the bilateral pelvis followed by a boost dose of 1400 cGy in 7 fractions to the left pelvic mass. Thus, the total radiation dose to the gross tumour in the left pelvis was 4460 cGy in 24 fractions. Beta-HCG showed a significant decline during the radiotherapy (1.4 on November 9,2005$)$, and returned to normal $(<0.5)$ at the end of the radiotherapy.

Since he completed the salvage radiotherapy, he has remained free of relapse. As of July 20, 2009, his beta-HCG and alpha-fetoprotein were normal and there was no evidence of relapse on the CT scan of the chest, abdomen and pelvis (Fig. 4).

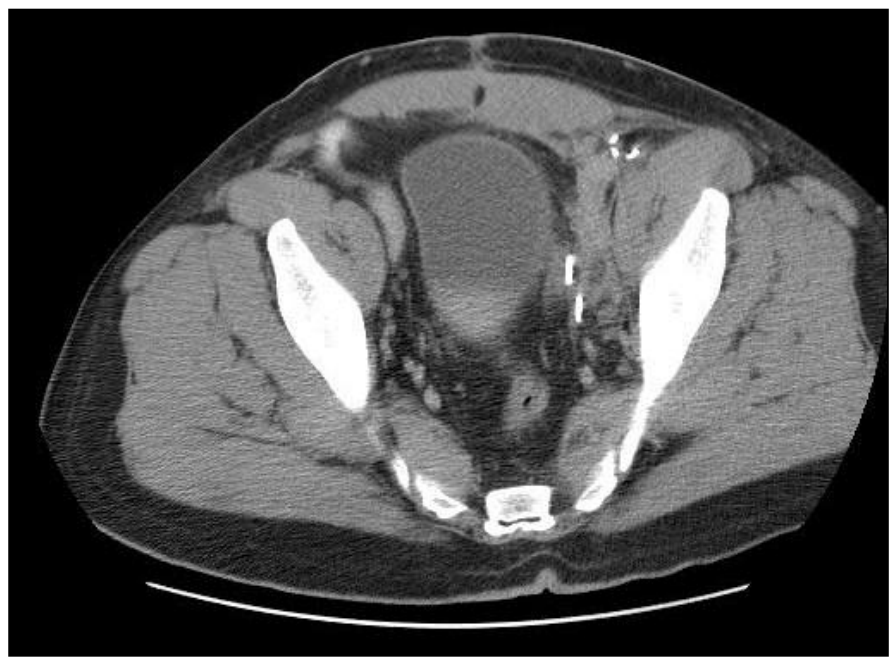

Fig. 2 Post surgery for the residual left pelvic mass in June 2005.

\section{Discussion}

The patient in this case presented with bulky stage IIC disease $(>5 \mathrm{~cm})$. Given a relatively large pelvic lymphadenopathy, he was treated primary chemotherapy consisting of 3 cycles of BEP as a standard approach. A residual mass after primary chemotherapy for bulky stage II or stage III seminoma is not uncommon, as demonstrated in this case. Management options for the residual mass include surgical resection and observation. Given a sizable residual mass in our case, surgery was undertaken, as suggested by others, ${ }^{1,2}$ and the pathology revealed only necrotic tissue. The patient relapsed, however, with an enlarging pelvic mass in a short period of time, associated with elevated beta-HCG.

The management approach for recurrent seminoma relapsed within 2 years of receiving primary chemotherapy usually involves salvage chemotherapy. The National Comprehensive Cancer Network guideline suggests salvage chemotherapy for a case like ours. ${ }^{3}$ Most salvage chemotherapy regimens incorporate ifosfamide and cisplatin combined with a third agent. VelP (vinblastine, ifosfamide and cisplatin) and TIP (paclitaxel, ifosfamide and cisplatin) are the 2 most common, standard-dose salvage regimens. Salvage chemotherapy is a prudent approach for patients relapsed with the involvement of both the supra- and the infra-diaphragmatic sites or distant organs. Should this salvage chemotherapy approach be adopted uniformly, however, for bulky stage IIC seminoma whose relapse is limited to an infradiaphragmatic nodal site?

There has been no literature describing a potential role of radiotherapy as salvage treatment for recurrent disease after primary chemotherapy for stage IIC seminoma. Gholam and colleagues reported a retrospective study examining treatment results and prognostic factors for survival after a firstline cisplatin-based chemotherapy for advanced seminoma. ${ }^{4}$ In this series, 31 (21\%) of 145 patients had recurrent disease 




Fig. 3. An enlarging left pelvic mass in October 2005.

after a first-line cisplatin-based chemotherapy. Of these 31 relapsed patients, 12 were treated with a multimodality regimen including chemotherapy, surgery and/or radiotherapy; the remaining patients were treated with salvage chemotherapy either alone or with autologous stem cell transplantation. Of the 12 patients treated with a multimodality regimen, 11 died of seminoma and only 1 one remained alive with no further relapse. All of these 12 patients had extensive tumour recurrence involving multiple organs at the time of the salvage therapy. This series did not specifically describe any single case that was salvaged by radiotherapy alone. Other available literature addressed the management issue related to a residual mass after chemotherapy, ${ }^{1,2}$ or discussed a limited benefit of routine administration of radiotherapy for a post-chemotherapy residual mass. ${ }^{5}$ No published series specifically examined a potential role of radiotherapy as salvage treatment for recurrent seminoma following primary chemotherapy, which is the main focus of our case report.

Our case demonstrates that radiotherapy can be a viable salvage treatment option for a recurrent disease following primary chemotherapy for a bulky stage IIC disease, provided that the recurrent disease is confined to the infradiaphragmatic site and that radiotherapy can be delivered without any major toxicity to critical organs, such as the kidney. The rationales for choosing radiotherapy instead of second-line chemotherapy are multifactorial: (1) Seminoma is very sensitive to radiotherapy, which implies a high probability of achieving loco-regional tumour control with radiotherapy. (2) The morbidity of radiotherapy is usually minor and generally less severe than second-line salvage chemotherapy. (3) Salvage chemotherapy will still be available if there is tumour recurrence after radiotherapy. Potential disadvantages of using radiotherapy as an alternative to second-line salvage chemotherapy can be also several: (1) Seminoma relapsing in the infradiaphragmatic site after primary chemotherapy is at risk of developing distant metastasis. Given

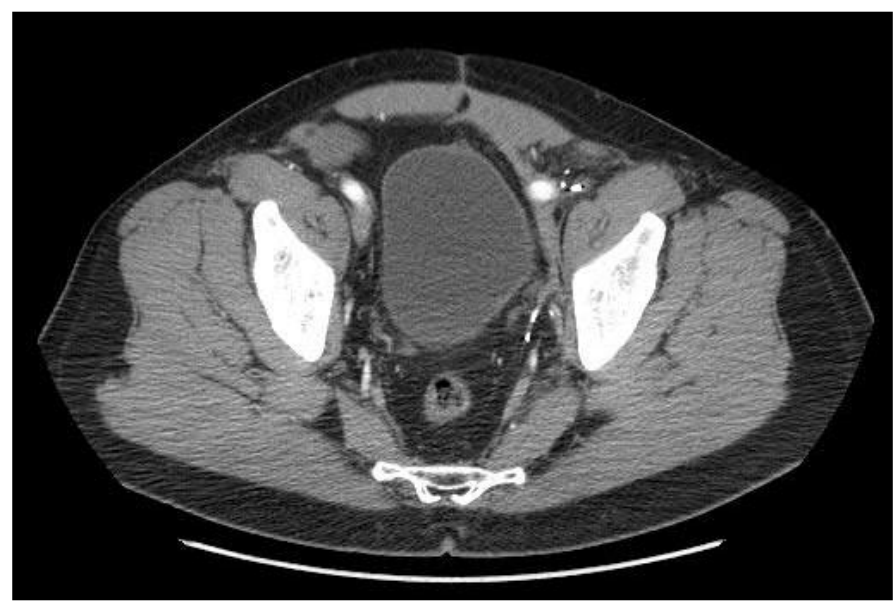

Fig. 4. Free of relapse at 3.7 years post-salvage radiotherapy in July 2009.

that radiotherapy is efficacious only to the area where it is administered, it can not address occult distant metastasis that may be already present at the time of relapse. (2) Because the irradiated volume is generally large in a salvage setting, radiotherapy can potentially compromise bone marrow reserve, which may become an important factor if the patient needs salvage chemotherapy in the future. (3) If a surgical resection is necessary for a residual mass, it can be more challenging after radiotherapy than after chemotherapy, since radiotherapy can cause fibrosis of irradiated tissues. (4) Radiotherapy may carry a higher risk of a second malignancy as a late morbidity, compared with second-line chemotherapy. In our case, salvage radiotherapy encompassed not only the relapsed nodal disease in the pelvis, but also the retroperitoneal lymphatic chain to address any occult nodal metastasis that might be present beyond the pelvis. The patient underwent the radiotherapy without any major acute morbidity including no adverse effect on blood counts. Also, with a follow-up of 3.7 years post-radiotherapy, he has not experienced any late radiation morbidity. Based on the case illustrated in this report, we suggest that radiotherapy should be considered as a management option for a patient with recurrent seminoma post primary chemotherapy for bulky stage IIC seminoma in which relapsed disease is limited to an infradiaphragmatic nodal site.

This case presents several other clinical and management facets that are worthy of discussion. First, the site of nodal metastasis in this case was in the pelvis, instead of the retroperitoneum where the testicular lymph typically drains into. This aberrant nodal metastasis at presentation is likely due to the patient's previous left inguinal hernia repair that could have disrupted the lymphatic drainage and redirected it to the iliac nodes. Second, this case confirms the current understanding that human chorionic gonadotropin can be elevated in seminoma and that it can a useful marker for the follow-up of seminoma. In our case, there 
Choo et al.

was a good correlation of serum beta-HCG with seminoma recurrence and its response to therapeutic interventions. Third, should the left pelvic mass, which manifested after a surgical resection of post-chemotherapy residual mass, have been biopsied for a definitive histologic diagnosis? We decided not to pursue a biopsy in this case because the relapse occurred shortly after the primary chemotherapy with no elevation of alpha-fetoprotein. This clinical picture was more consistent with the recurrence of the original tumour than the development of non-seminomatous germ cell tumour. Another possible explanation for the left pelvic mass is a non-malignant process, such as a lymphocele or postoperative change. However, the possibility that the left pelvic mass would be due to a lymphocele or postoperative change is very remote in our case, since a benign process, such as a lymphocele or postoperative change, usually shows a regression over time, as opposed to a progression shown in our case. Another test that might have strengthened the diagnosis of tumour recurrence is a positron emission tomography, which was not performed in our case. Fourth, the radiation dose used in our case was higher than that used in a primary radiotherapy setting for stage II seminoma. We deliberately chose a higher radiation dose for the following 2 reasons. Firstly, a higher radiation dose might be required for the possibility of radio-resistant tumour subpopulation, given that the tumour had already demonstrated a biologically aggressive potential, evidenced by a progression after a cisplatin-based chemotherapy. Secondly, since radiotherapy is a one-time therapeutic intervention with almost no chance of repeating it in future, we chose a high radiation dose that could provide a maximum chance of eradicating the tumour, but at the same time, a dose not too excessive to cause an increased risk of late radiation morbidity, such as small bowel obstruction.

\section{Conclusion}

The current management paradigm for recurrent seminoma relapsed within 2 years of receiving primary chemotherapy usually calls for salvage second-line chemotherapy. However, in the setting of bulky stage IIC seminoma whose relapse is limited to an infradiaphragmatic nodal site, radiotherapy can be a potentially viable salvage treatment option. Further evaluation is needed to confirm the potential role of radiotherapy as salvage treatment for recurrent seminoma post-primary chemotherapy.

*Department of Radiation Oncology, Mayo Clinic, Rochester, MN; †Department of Medical Oncology, Mayo Clinic, Rochester, MN; „Department of Urology, Mayo Clinic, Rochester, MN

Competing interests: None declared.

This paper has been peer-reviewed.

\section{References}

1. Herr HW, Sheinfeld J, Puc HS, et al. Surgery for a post-chemotherapy residual mass in seminoma. J Urol 1997; 157:860-2.

2. Puc HS, Heelan R, Mazumdar M, et al. Management of residual mass in advanced seminoma: results and recommendations from the Memorial Sloan-Kettering Cancer Center. J Clin Oncol 1996;14:454-60.

3. National Comprehensive Cancer Network. NCCN Clinical Practice Guidelines in Oncology, Testicular Cancer. http://www.nccn.org/professionals/physician_gls/PDF/testicular.pdf (Accessed August 6, 2010).

4. Gholam D, Fizazi K, Terrier-Lacombe MJ, et al. Advanced seminoma-treatment results and prognostic factors for survival after first-line, cisplatin-based chemotherapy and for patients with recurrent disease: a single-institution experience in 145 patients. Cancer 2003;98:745-52.

5. Duchesne GM, Stenning SP, Aass N, et al. Radiotherapy after chemotherapy for metastatic seminoma - a diminishing role. MRC Testicular Tumour Working Party. Eur J Cancer 1997;33:829-35.

Correspondence: Dr. Richard Choo, Mayo Clinic, Department of Radiation Oncology, 200 First St., SW, Rochester, MN 55905; fax: 507-284-0079; Choo.@@mayo.edu 